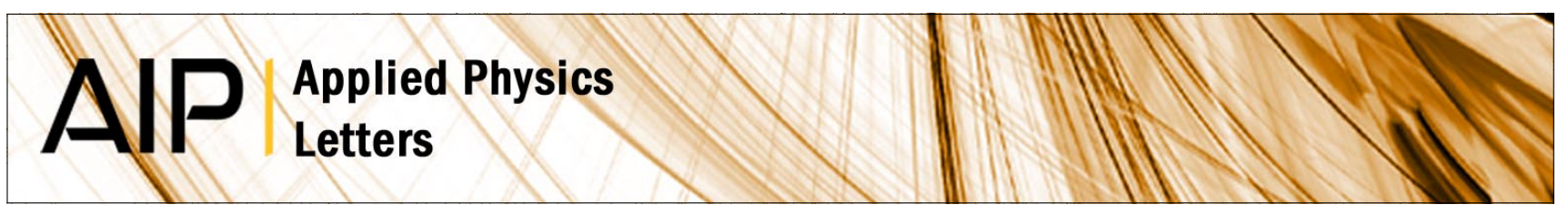

\title{
Boron-oxygen defect imaging in p-type Czochralski silicon
}

S. Y. Lim, F. E. Rougieux, and D. Macdonald

Citation: Appl. Phys. Lett. 103, 092105 (2013); doi: 10.1063/1.4819096

View online: http://dx.doi.org/10.1063/1.4819096

View Table of Contents: http://apl.aip.org/resource/1/APPLAB/v103/i9

Published by the AIP Publishing LLC.

Additional information on Appl. Phys. Lett.

Journal Homepage: http://apl.aip.org/

Journal Information: http://apl.aip.org/about/about_the_journal

Top downloads: http://apl.aip.org/features/most_downloaded

Information for Authors: http://apl.aip.org/authors

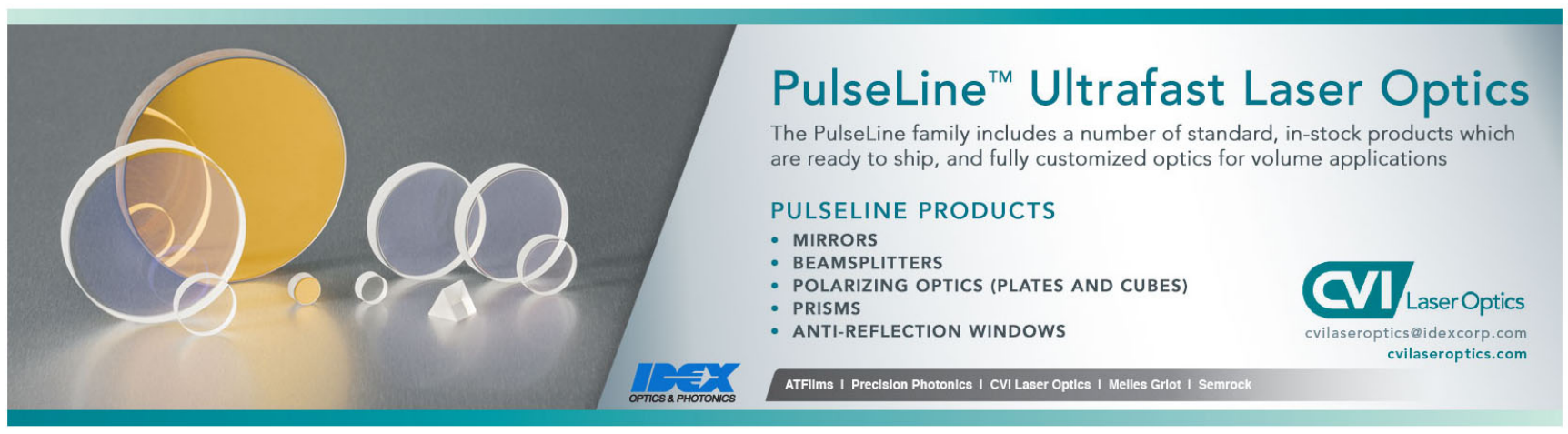




\title{
Boron-oxygen defect imaging in p-type Czochralski silicon
}

\author{
S. Y. Lim, F. E. Rougieux, and D. Macdonald \\ Research School of Engineering, College of Engineering and Computer Science, The Australian National \\ University, Canberra, ACT 0200, Australia
}

(Received 10 July 2013; accepted 2 August 2013; published online 27 August 2013)

\begin{abstract}
In this work, we demonstrate an accurate method for determining the effective boron-oxygen (BO) related defect density on Czochralski-grown silicon wafers using photoluminescence imaging. Furthermore, by combining a recently developed dopant density imaging technique and microscopic Fourier transform infrared spectroscopy measurements of the local interstitial oxygen concentration $\left[\mathrm{O}_{i}\right]$, the $\mathrm{BO}$-related defect density, $\left[\mathrm{O}_{i}\right]$, and the boron dopant density from the same wafer were determined, all with a spatial resolution of $160 \mu \mathrm{m}$. The results clearly confirm the established dependencies of the BO-related defect density on $\left[\mathrm{O}_{i}\right]$ and the boron dopant density and demonstrate a powerful technique for studying this important defect. (C) 2013 AIP Publishing LLC. [http://dx.doi.org/10.1063/1.4819096]
\end{abstract}

The effect of the boron-oxygen (BO) related defect on the performance of silicon solar cells continues to be an active area of research. However, the precise chemical composition of the defect and a detailed understanding of its transformations under illumination and annealing still remain unclear. ${ }^{1,2}$ Bothe et al. ${ }^{1}$ have shown that the extent of formation of the defect is linearly related to the boron dopant density $N_{A}$ (at least in non-compensated silicon) and quadratically related to the interstitial oxygen concentration $\left[\mathrm{O}_{i}\right]$. However, in previous studies, a complication is sometimes caused by the fact that different thermal histories and growth conditions can potentially affect the BO defect density. This paper aims to test the established relationships between the BO defect density, $N_{A}$, and $\left[\mathrm{O}_{i}\right]$ on a single wafer, ensuring that both the assumptions of similar growth conditions and thermal history are valid. This is performed by combining high resolution measurements of all three parameters on a single sample which contains significant variations in the oxygen and boron concentrations. In the process, we also demonstrate a method for accurate $\mathrm{BO}$ defect imaging using photoluminescence, which takes proper account of the impact of varying injection levels.

The determination of the effective BO defect density $N_{t}$ has conventionally been accomplished by using the quasisteady-state photoconductance (QSSPC) minority carrier lifetime measurement tool, defined by $N_{t}=1 / \tau_{\text {degraded }}-1 / \tau_{\text {annealed, }}$, where $\tau_{\text {degraded }}$ is the degraded lifetime measured after lightinduced degradation, and $\tau_{\text {annealed }}$ the lifetime of the sample after the boron oxygen defect is de-activated, ${ }^{3}$ and both are measured at the same excess carrier density. It is common practice to choose an excess carrier density of $0.1 \times N_{A}$, in which case the relative defect concentration is referred to as $N_{t}{ }^{*}$. This provides a measurement standard, allows comparison between different resistivities, and gives a reasonable compromise between the impact of Auger recombination at higher injection, and minority carrier trapping effects at lower injection, which impact QSSPC measurements. ${ }^{3}$ The recent development of a photoluminescence based lifetime imaging tool (PL), which is applicable under low injection conditions and unaffected by various experimental artefacts caused by minority trapping and depletion region modulation, ${ }^{4}$ has enabled more in-depth studies by allowing high resolution spatial measurement of the defect, as reported previously. ${ }^{5,6}$ In principle, the use of PL imaging at low injection allows the BO defect density to be determined in the injection range in which it has no injection dependence, leading to a more robust determination of the effective defect density $N_{t}$.

However, the calculation of the $\mathrm{BO}$ defect density $N_{t}$ using pairs of PL images obtained with a constant generation rate before and after activation has its own potential problems, due to the changing injection level between the two images. This can be seen in the modelled carrier lifetime versus the excess carrier density graph shown in Fig. 1. This simulation is based on 808-nm monochromatic incident light and applying Klaassen's mobility model, using the established energy level and capture cross section ratio for the BO defect to model its injection dependence. ${ }^{2,3,7}$ The boron concentration $N_{A}$ is taken as $3.5 \times 10^{16} \mathrm{~cm}^{-3}$, the same as the experimental sample in this work. The angled lines represent various constant generation rates. Where these lines intersect the lifetime curves represents the lifetime measured for a given generation rate. It can be seen that the points of intersection are at significantly different excess carrier densities before and after activation of the defect, if a single generation rate is used. Since the lifetime increases with injection level in the activated state, this leads to an underestimation of the lifetime (shown as $\Delta \tau$ on the plot), which in turn results in an overestimation of the BO-related defect density. This may be avoided in principle by calculating the impact of the injection dependence at the reduced injection level, based on the knowledge of the capture cross section ratio, ${ }^{5}$ but even this approach may be inaccurate when other injection-dependent recombination centres are present. To avoid such discrepancies in BO-related defect imaging, in this work we developed a multiple imaging approach in which the degraded lifetime can be determined directly at the desired injection level via a numerical interpolation procedure based on multiple images acquired at different generation rates, as indicated in Fig. 1. Note, however, that even if a low injection level is chosen, e.g., this work, for which the BO defect has no injection dependence, it is still prudent to extract the lifetimes at the same excess carrier density, since 


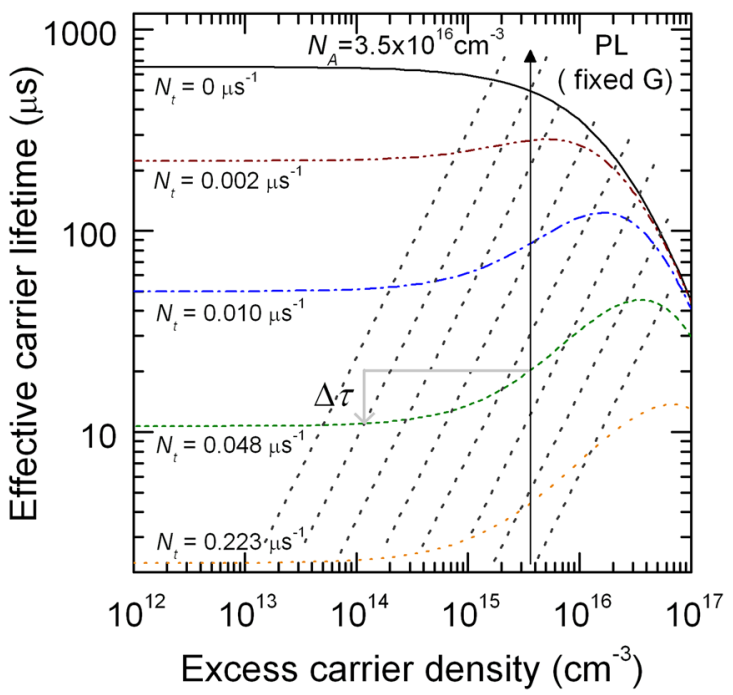

FIG. 1. Numerical simulation of bulk lifetime versus excess carrier density. The solid vertical arrow shows the standard measurement condition at $0.1 \times N_{A}$. Discrepancy in lifetime is introduced when a constant generation measurement is used (as represented by the angled arrow), resulting in an underestimation of the degraded lifetime.

other recombination channels may still impart some injection dependence on the measured data, which could otherwise affect the results.

PL images in this work were obtained with a BT imaging LIS-R1 instrument, ${ }^{4}$ described in detail elsewhere. ${ }^{8}$ The $163 \mu \mathrm{m}$ thick sample used in this work was a cleaved quarter section of a $0.47 \Omega \mathrm{cm}, 155 \times 155 \mathrm{~mm}^{2}$ pseudosquare B-doped, $\langle 100\rangle$ oriented Czochralski-grown silicon wafer. Surface passivation was achieved by depositing a SiN film at $400{ }^{\circ} \mathrm{C}$ on both surfaces using Plasma-Enhanced Chemical-Vapour Deposition (PECVD).

Calibrated minority carrier lifetimes were measured with the PL imaging tool and were performed on the silicon sample after a $30 \mathrm{~min}$ annealing at $200{ }^{\circ} \mathrm{C}$ in the dark to enable complete BO-related deactivation followed by another $30 \mathrm{~min}$ in the dark at room temperature to allow complete pairing of any dissolved iron with boron in the sample. 9,10 The minority carrier lifetime was measured again after the sample was exposed to illumination under a halogen lamp with an intensity equivalent to approximately one-tenth of 1 sun to activate the BO-related defect for intervals from $20 \mathrm{~s}$ to 2 days. Prior to each PL image, the sample was left in the dark for $30 \mathrm{~min}$ for complete FeB pairing. PL measurements were obtained at laser flux intensities between $1.0 \times 10^{17}$ and $2.2 \times 10^{17}$ photons $\mathrm{cm}^{-2} \mathrm{~s}^{-1}$ for an acquisition time of $0.2 \mathrm{~s}$, which is sufficiently short to avoid significant breaking of any iron boron pairs during the measurement. The lifetimes for determining the effective $\mathrm{BO}$ defect concentration were extracted at a fixed excess carrier density of $0.001 \times N_{A}$, which corresponds to the range of $\Delta \mathrm{n}$ from $2.4 \times 10^{13}$ to $4.7 \times 10^{13} \mathrm{~cm}^{-3}$, by interpolating the multiple images taken at each condition.

The dopant density measurement was performed on a cleaved sister quarter of the same material after a surface treatment was performed to achieve the surface limited conditions, where dopant density images can be obtained through the PL imaging process, as described in detail elsewhere. ${ }^{8}$

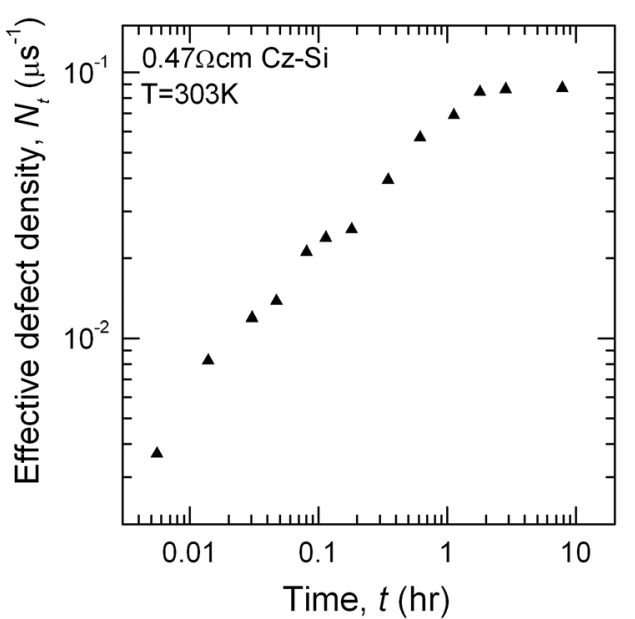

FIG. 2. Sample averaged effective defect density as a function of time under 0.1 sun illumination.

This surface treatment involves roughening of a sample surface with hot water at $75^{\circ} \mathrm{C}$ for $5 \mathrm{~min}$. This PL image is measured with a laser flux intensity of $1.6 \times 10^{17}$ photons $\mathrm{cm}^{-2} \mathrm{~s}^{-1}$ for an acquisition time of $20 \mathrm{~s}$, and the dopant density was calibrated based on an average resistivity value measured with dark conductance. All data presented here based on PL images are first subjected to deconvolution with a point-spread-function (PSF) to reduce the impact of lightscattering effects in the detector. ${ }^{11}$ A line scan of the interstitial oxygen density was measured on the same sample from centre to edge with a step size $160 \mu \mathrm{m}$, after it was polished by silicon etching solution. The measurements were performed with a Bruker microscopic FTIR system at a resolution of $4 \mathrm{~cm}^{-1}$ and a laser spot size of $10 \mu \mathrm{m}$. The $\left[\mathrm{O}_{i}\right]$ measurements were based on the ASTM standard F121-80.

The measured averaged effective defect density, $N_{t}$, from the whole quarter wafer as a function of time during the light-induced degradation process is shown in Fig. 2. The result indicates that the defect has reached saturation after approximately $3 \mathrm{~h}$ (around $10^{4} \mathrm{~s}$ ) of light exposure. Fig. 3 shows the BO-related defect images after exposure to

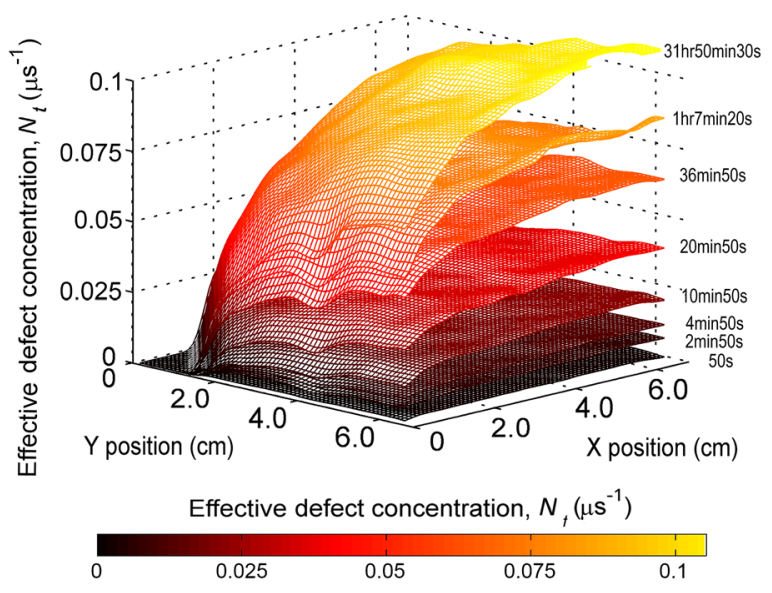

FIG. 3. Time resolved images of $N_{t}$ defect density after different illumination times from $50 \mathrm{~s}$ to $31 \mathrm{~h} 50 \mathrm{~min} 30 \mathrm{~s}$ showing inhomogeneous BO-related defect density distribution, with higher concentration in the central region of the wafer. 

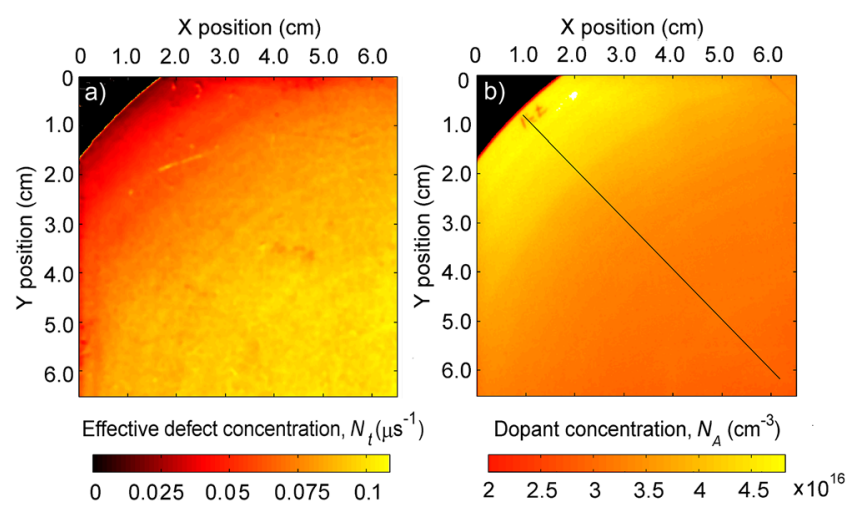

FIG. 4. (a) Effective defect density $N_{t}$ after complete activation and (b) dopant density images, showing opposing profiles, revealing the stronger impact of $\left[\mathrm{O}_{i}\right]$ on the $\mathrm{BO}$ defect generation. FTIR measurements are performed on the line scan shown in (b).

illumination for different times $(50 \mathrm{~s}, 2 \min 50 \mathrm{~s}, 4 \min 50 \mathrm{~s}$, $10 \min 50 \mathrm{~s}, \quad 20 \min 50 \mathrm{~s}, \quad 36 \min 50 \mathrm{~s}, \quad 1 \mathrm{hr} 7 \min 20 \mathrm{~s}$, and $31 \mathrm{hr} 50 \mathrm{~min} 30 \mathrm{~s})$. The defect density is non-uniformly distributed and higher in the central region. The saturated effective defect density image is shown in Fig. 4(a). Fig. 4(b) shows the corresponding surface limited dopant density image, after being calibrated with dark conductance measurements. It can be observed that the dopant density is higher at the edge and reduces by about $50 \%$ in the central region. This is the characteristic of a crystallisation interface that is concave toward the melt during crystal growth. The appearance of the counter correlation between these two images reveals that the effect of the boron concentration on the BOrelated defect density is counteracted by a stronger influence of the interstitial oxygen concentration, as confirmed below. Fig. 5 shows the FTIR spectra measured from the wafer edge to the centre at $8 \mathrm{~mm}$ intervals along the line scan of Fig. 4(b). The spectra show increasingly higher $\left[\mathrm{O}_{i}\right]$ towards the centre of the sample, as evidenced by the drop in transmittance near $1100 \mathrm{~cm}^{-1}$. Also, an increase in free carrier absorption can be seen from edge to centre of the wafer, reflecting a decrease in dopant density towards the centre of the wafer, confirming the $N_{A}$ profile previously obtained via

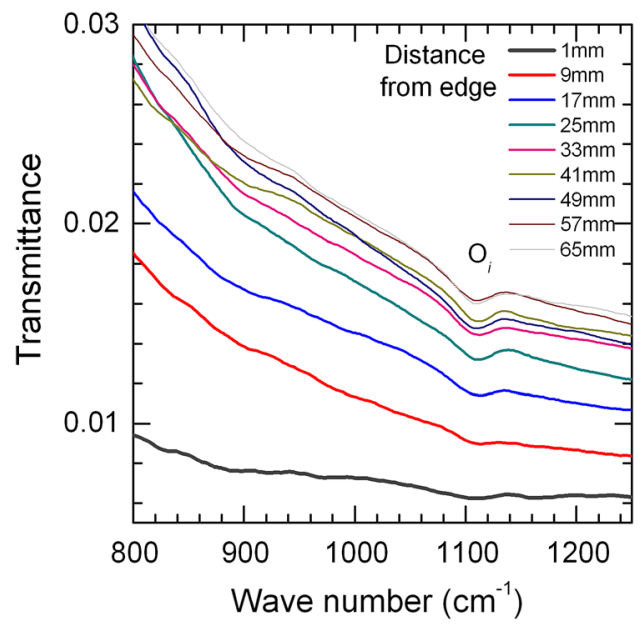

FIG. 5. Measured FTIR spectra along the line scan of Fig. 4(b), showing higher $\left[\mathrm{O}_{i}\right]$ in the centre region of the sample, where the free carrier absorption is found to be lower indicating a decrease in dopant density.

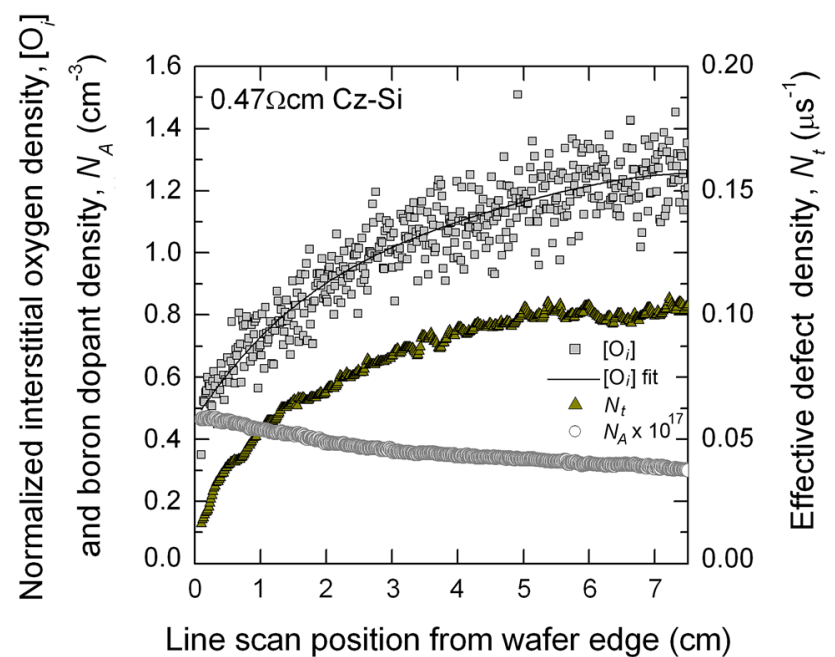

FIG. 6. (a) Measured $N_{t}, N_{A}$, and $\left[\mathrm{O}_{i}\right]$ densities, reflecting the dominant influence of $\left[\mathrm{O}_{i}\right]$ on the $N_{t}$.

the surface-limited PL image. The $\left[\mathrm{O}_{i}\right]$ derived from the FTIR spectra with $160 \mu \mathrm{m}$ spatial resolution are shown in Fig. 6. A fit to the data is also shown. The uncertainty in the $\left[\mathrm{O}_{i}\right]$ data is approximately $10 \%$. In the figure, line scans of the measured $N_{A}$ and BO-related defect density, $N_{t}$ obtained from the PL-based images above, are also shown. Clearly the $\left[\mathrm{O}_{i}\right]$ and the BO-related defect density $N_{t}$ data follow the same trend, reflecting the dominant influence of the $\left[\mathrm{O}_{i}\right]$ on the BO-related defect density.

The measured data of $N_{A},\left[\mathrm{O}_{i}\right]$, and $N_{t}$ can be used to derive the relationship between the three factors. To investigate this, the ratio of the measured parameters $N_{t} /\left(\left[\mathrm{O}_{i}\right]_{\text {(measured })}{ }^{\mathrm{a}}\right.$ $\times N_{A \text { (measured })}$ ) ) along the line scan of Fig. 4 using different values for the exponents $a$ and $b$ is performed, and the results are shown in Fig. 7 (normalized to the value near the centre of the wafer). Within the uncertainties, a constant value of close to unity is yielded when $a=2$ and $b=1$. Additionally, an assessment of the uncertainty in the values of $a$ and $b$ can be made by varying these parameters, until the data fall significantly outside the error bars. The result yields a range of 1.7-2.2 for the power in $\left[\mathrm{O}_{i}\right]$ and $0.7-1.4$ for the power in $N_{A}$.

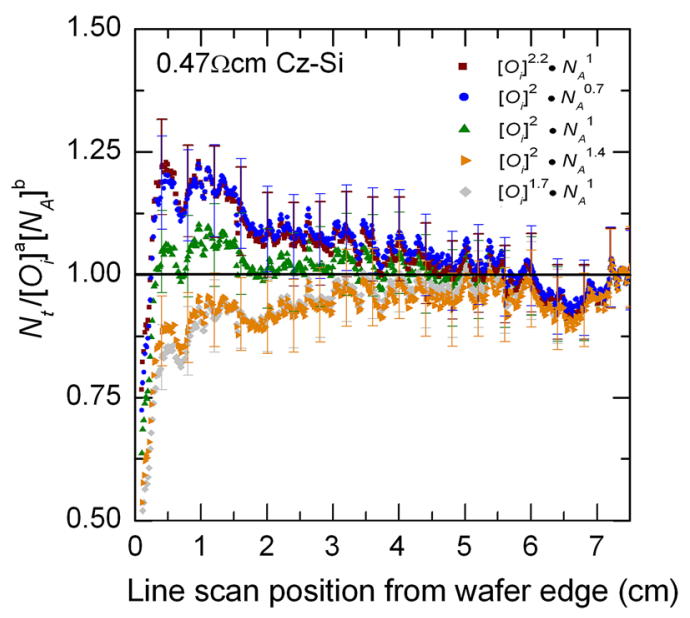

FIG. 7. Linescan of the normalized ratio of $N_{t} /\left[\left[\mathrm{O}_{i}\right]^{a} \times N_{A}{ }^{b}\right]$ for a range of values for $a$ and $b$. Values close to unity are obtained for $N_{t} \propto N_{A}{ }^{1} \times\left[\mathrm{O}_{i}\right]^{2}$. 
This confirms, in an unambiguous way, the established dependence between these variables, for example, those reported by Bothe in which the powers were determined to be $1.9 \pm 0.1$ and $1.05 \pm 0.05$, respectively. ${ }^{1}$

In conclusion, we have demonstrated an accurate method of imaging boron oxygen defects using the photoluminescence imaging technique by adopting a multiple imaging procedure. This approach can in principle be applied at either moderate injection levels, where there is still some injection dependence of the BO defect, or under low injection conditions where the defect has no injection dependence, although other recombination channels still may be present. Further, by incorporating images of the dopant density based on surface-limited PL images, and line scans of the interstitial oxygen concentration using microscopic FTIR spectroscopy from the same wafer, we confirm the linear influence of the boron dopant density and the quadratic influence of the interstitial oxygen on the BO-related defect density. This method can also, in principle, be applied to compensated wafers to gain further insights into the behaviour of the defect in the presence of compensating dopants.
This work was supported by the Australian Research Council (ARC) Future Fellowships program and the Australian Renewable Energy Agency (ARENA) fellowships program. The authors acknowledge Thorsten Trupke for helpful discussions.

${ }^{1}$ K. Bothe and J. Schmidt, J. Appl. Phys. 99, 013701 (2006).

${ }^{2}$ S. Rein and S. W. Glunz, Appl. Phys. Lett. 82, 1054 (2003).

${ }^{3}$ K. Bothe, R. Sinton, and J. Schmidt, Prog. Photovoltaics 13, 287-296 (2005).

${ }^{4}$ T. Trupke, R. A. Bardos, M. C. Schubert, and W. Warta, Appl. Phys. Lett. 89, 044107 (2006).

${ }^{5}$ M. C. Schubert, H. Habernicht, and W. Warta, IEEE J. Photovoltaics 1, 168 (2011).

${ }^{6}$ M. Wilson, P. Edelman, A. Savtchouk, J. D'Amico, A. Findlay, and J. Lagowski, J. Electron. Mater. 39, 642-647 (2010).

${ }^{7}$ A. Cuevas, Energy Proc. 8, 94-99 (2011).

${ }^{8}$ S. Y. Lim, M. Forster, X. Zhang, J. Holtkamp, M. Schubert, A. Cuevas, and D. Macdonald, IEEE J. Photovoltaics 3, 649 (2012).

${ }^{9}$ W. Wijaranakula, J. Electrochem. Soc. 140, 275-281 (1993).

${ }^{10}$ D. Macdonald, T. Roth, P. N. K. Deenapanray, K. Bothe, P. Pohl, and J. Schmidt, J. Appl. Phys. 98, 083509 (2005).

${ }^{11}$ D. Walter, A. Liu, E. Franklin, D. Macdonald, B. Mitchell, and T. Trupke, in Proceedings of the 38th IEEE Photovoltaic Specialists Conference, Texas, 2012. 